\title{
TRACING AND COMPARING SERUM, SPECIFIC, BONE BIOMARKERS IN PATIENTS WITH SECONDARY HYPERPARATHYROIDISM
}

\author{
Svetla Staykova ${ }^{1}$, Yana Bocheva ${ }^{2}$ \\ ${ }^{1}$ Department of Internal Diseases, Faculty of Medicine, Medical University of Varna \\ ${ }^{2}$ Department of General Medicine and Clinical Laboratory, Faculty of Medicine, \\ Medical University of Varna
}

\begin{abstract}
Bone and mineral disorders (BMDs) in chronic kidney disease (CKD) are increasingly being studied, and prophylaxis and treatment are conducted, but they still remain one of the most severe systemic illnesses in patients with CKD.

In patients with end-stage CKD and secondary hyperparathyroidism, accompanying metabolic disorders of calcium and phosphorus homeostasis may lead to pathological changes in bone and blood vessels, which increase the risk of bone fractures and cardiovascular (CV) events. High levels of parathyroid hormone (PTH), calcium and phosphorus are associated with increased morbidity and mortality in dialysis patients.

Dialysis treatment is a renal replacement method that continues the life of patients with CKD, temporarily improving existing bone pathology, but it more often accelerates its progression. Therefore, the symptoms, developmental and complications of BMD-CKD are demonstrated and manifested in patients with extracorporeal treatment.

Treatment of BMD requires constant monitoring of Ca-P exchange, PTH, serum of Vitamin D levels and the protein bone markers - osteocalcin, bone alkaline phosphatase.

Despite the systemic use of active metabolites of vitamin $D$, phosphate binders and calcimimetics, in many patients with secondary hyperparathyroidsm, inadequate biochemical control has been observed.

In the Dialysis Clinic at St. Marina University Hospital, Varna, two groups of patients on hemodialysis (HD) and with CKD - 2/3 stage with secondary hyperparathyroidism, were followed and had their serum biomarker levels compared - PTH, bone alkaline phosphatase (BAP), osteocalcin, and vitamin D. The results showed statistically significant differences between the two groups in the investigated serum levels of the indicators.
\end{abstract}

Keywords: secondary hyperparathyroidism, biomarkers, hemodialysis treatment, pre-dialysis stage

Address for correspondence:

Svetla Staykova

Faculty of Medicine

Medical University of Varna

55 Marin Drinov St

9002 Varna

e-mail: svetlastaykova@abv.bg

Received: November 28, 2017

Accepted: March 30, 2018

\section{INTRODUCTION}

In patients with end-stage chronic kidney disease (CKD) and secondary hyperparathyroidism, accompanying metabolic disorders of calcium and phosphorus homeostasis may result in pathological changes in bone tissue and vessels that increase the risk of bone fractures and cardiovascular disorders (1). High levels of parathyroid hormone (PTH), calcium and phosphorus are associated with increased 
morbidity and mortality in patients with secondary hyperparathyroidism due to dialysis. The available options for treatment of bone mineral disorders do not always provide adequate control of $\mathrm{PTH}$, calcium and phosphorus levels, and are often associated with mismatch and high incidence of discontinuation (2-5).

CKD affects all key steps in vitamin D metabolism, namely: production, activation and degradation of vitamin $\mathrm{D}$ and its metabolites at 25-hydroxyvita$\min \mathrm{D}(25(\mathrm{OH}) \mathrm{D})$ and low levels of 1,25 dihydroxyvitamin $\mathrm{D}(1,25(\mathrm{OH}) 2 \mathrm{D})(6)$. The loss of vitamin $\mathrm{D}$ in urine is used as a marker for vitamin $\mathrm{D}$ levels in patients with high proteinuria. However, it can be expected that the decrease in vitamin $\mathrm{D}$ would initially reduce the levels of $1,25(\mathrm{OH}) 2 \mathrm{D}$, and subsequently those of the fibroblast growth factor-23 (FGF-23).

The results of a recent study of patients categorized in phenotypes based on PTH, calcium and phosphate levels demonstrated that phenotypically elevated levels of PTH, phosphate and calcium carry a greater risk of death or hospitalization associated with a cardiovascular event in comparison to phenotypes where all three parameters are within the target values $(7,8)$.

The use of vitamin $\mathrm{D}$ receptor activator in the pre-dialysis stage has an inhibitory effect on the elevation in the serum levels of PTH at the time of dialysis initiation.

\section{MATERIALS AND METHODS}

The serum level of biomarkers - PTH, vit. D and osteocalcin, bone alkaline phosphatase (BAP) were monitored in two groups of patients - 28 men and 30 women on hemodialysis (HD) treatment at the Dialysis Clinic of St. Marina University Hospital, Varna; and 18 men and 29 women in pre-dialysis stage with CKD.

The following methods were used to screen iPTH, $25 \mathrm{OH}$ vitamin $\mathrm{D}$, osteocalcin and BAP OSTASE ${ }^{\oplus}$ t the LIAISON ${ }^{\circledR} 25 \mathrm{OH}^{2}$ Vitamin D TOTAL test, which applies a chemiluminescent immune test (CLIA) technology for the quantification of 25-hydroxyvitamin $\mathrm{D}$ and other hydroxylated metabolites of vitamin D in human serum; the LIAISON ${ }^{\circledR}$ Osteocalcin Test of DiaSorin, which is a single-step immunohistochemical assay (IHCA) designed to quantify the osteocalcin polypeptide in human serum.
The method for quantification of osteocalcin was a direct chemiluminescent immunoassay (CLIA). DiaSorin LIAISON ${ }^{\circledR}$ BAP OSTASE $^{\oplus}$ is a one-step supplemental immune hemolytic assay (IHLA) designed for quantification of BAP in human serum.

The precision of the LIAISON ${ }^{\circledR}$ assay was evaluated according to CLSI EP5-A2.

All blood samples for the biomarkers tested were taken at the beginning of the dialysis session in a "yellow" vacuum $-5 \mathrm{ml}$ of the blood haemolines before the anticoagulant was placed in the extracorporal system. The blood sample was centrifuged for 10 minutes at $3600 \mathrm{rpm}$ to coagulate and the serum was separated from the clot. Thus, the resulting serum was dispensed into 2 flasks - plastic vials of 1.25 $\mathrm{ml}$, and stored at up to $6^{\circ} \mathrm{C}$ or frozen $\left(-20^{\circ} \mathrm{C}\right.$ or less). A specialized package StatSoft Inc, USA, STATISTICA Manual (data analysis onl 0.0,system), Version 10.0, 2010 was used for statistical analysis of the data.

1. Descriptive statistical analysis - it showed a tabular frequency distribution of the examined signs, broken down by study groups, averages and standard deviations, 95\% confidence intervals of change of the mean values.

2. Student's test (t-criterion) for two independent samples was applied to detect a statistically significant difference in the mean of a factor in two patient groups.

3. Student's test (t-criterion) for two paired samples was applied to detect a statistically significant difference in the mean values of a factor before and after treatment.

\section{RESULTS AND DISCUSSION}

Osteocalcin is the major non-collagen protein in human bones. It is synthesized by the osteoblasts and the odontoblasts of bone tissue. The main part of the synthesized protein enters the extracellular bone matrix, which then mineralizes with the formation of new bone and the rest falls into the blood stream. Its values are increased in metabolic bone diseases with accelerated bone metabolism, such as osteoporosis, osteomalacia and rickets, hyperparathyroidism, renal osteodystrophy, thyrotoxicosis and acromegaly (Fig. 1). It has low values in cases of growth hormone deficiency, hypoparathyroidism, hypothyroidism (9). Therefore, there was a statistically signif- 


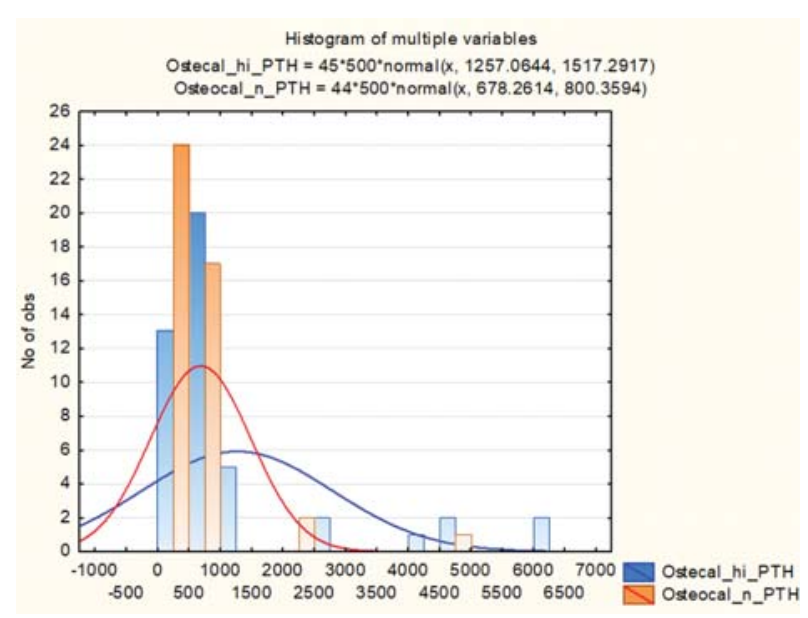

Fig. 1. Change of Osteocalcin in patients on HD and predialysis stage

icant difference in the osteocalcin mean values of patients in both groups.

\section{Kidney Disease}

Improving Global Outcomes (KDIGO) recommends maintaining PTH levels in the range of approximately 2 to 9 times the upper limit of normal (the proposed level of PTH from KDIGO corresponds to 120 to $660 \mathrm{pg} / \mathrm{mL}$ ), while calcium is maintained within the normal range and elevated levels of phosphate decreased to normal in patients with CKD on dialysis.

Our study showed a significant difference in the values of vit. $\mathrm{D}$ in the two groups of patients at the pre-dialysis and dialysis stage (Fig.2).

Vitamin D deficiency is associated with metabolic syndrome and obesity in HD patients. It is also

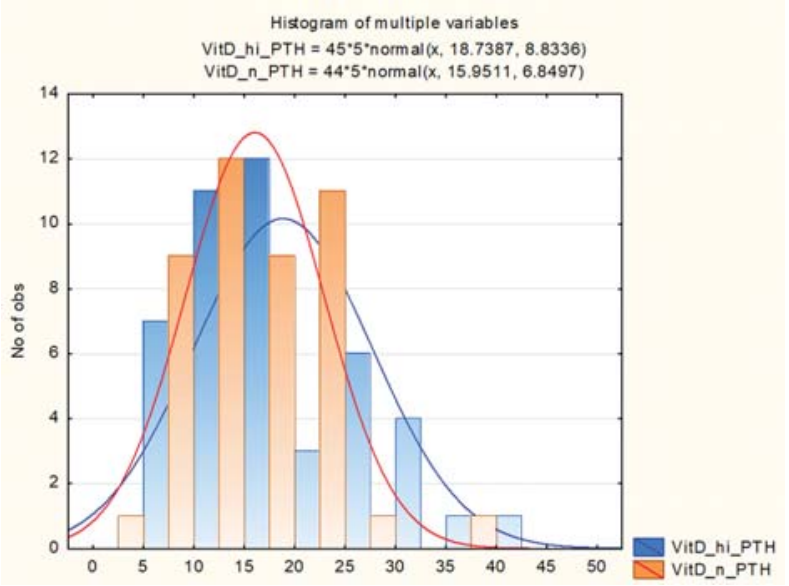

Fig. 2. Values of Vit D in both patient groups associated with insulin resistance, ventricular hypertrophy, atherosclerosis and vascular calcifications.

London et al. reported a feedback between arterial stiffness and serum 25(OH)D and 1,25(OH)2D levels in dialysis patients.

According to Boudville et al. the deficiency of $25(\mathrm{OH}) \mathrm{D}$ is associated with muscle weakness and decreases in dialysis patients with a curve and a maximum benefit in the range of 24 to $44 \mathrm{ng} / \mathrm{mL}$ of serum levels of $25(\mathrm{OH}) \mathrm{D}$. Vitamin $\mathrm{D}$ deficiency is associated with metabolic syndrome and obesity (10) in HD patients. In patients at the pre-diaysis stage, low levels of vitamin D are associated with cognitive impairment (11).

According to studies that report results from North America, Asia, Australia and New Zealand, approximately $50 \%$ of the patients there and over $30 \%$ of those in Europe have available biochemical evidence of secondary hyperparathyroidism (SHPT) defined as $\mathrm{PTH}>300 \mathrm{pg} / \mathrm{mL}$ (12).

SHPT may lead to hyperparathyroid bone disease - a common cause of bone and joint pain in dialysis patients, a reduction in bone mass, and an increase in the risk of bone fractures (13).

Phosphorus induces the secretion of PTH by three mechanisms: direct stimulation of the parathyroid glands; induction of hypocalcemia, increased activity of FGF-23 (resulting in inhibition of alpha-1 hydroxylase and lowering levels of 1,25(OH)2D).

Calcium and phosphorus metabolism disorders in the SHPT are thought to contribute to the calcification of soft tissues and the vascular system with $50 \%$ to $80 \%$ prevalence.

Hyperphosphatemia is one of the most common complication of CKD and, if not treated, leads to pruritus, bone pain, anemia, cardiovascular complications, disability, and reduced survival.The changes in PTH levels relative to age (Fig. 3) are shown in two groups of patients - pre-dialysis with CKD - 47 ( 35 women and 12 men) and patients on HD - 50 (28 women and $22 \mathrm{men}$ ), who are tracked dynamically at the Dialysis Clinic. A statistically significant difference between the two groups was seen with Paricalcitol at a dose of $1 \mathrm{mg} / \mathrm{day}$ (Tabl. 1).

Therefore, there was no statistically significant difference in the mean values of the patients in the two groups. 


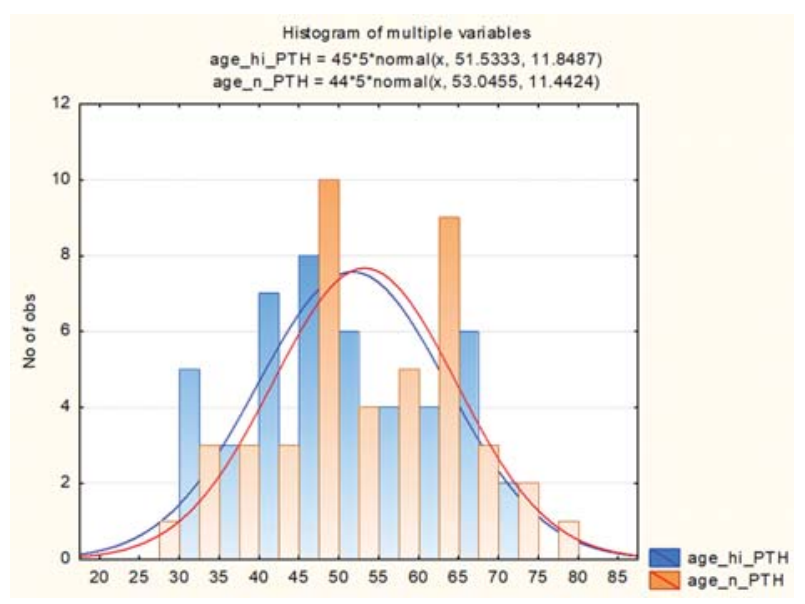

Fig. 3. Change of the PTH relative to age

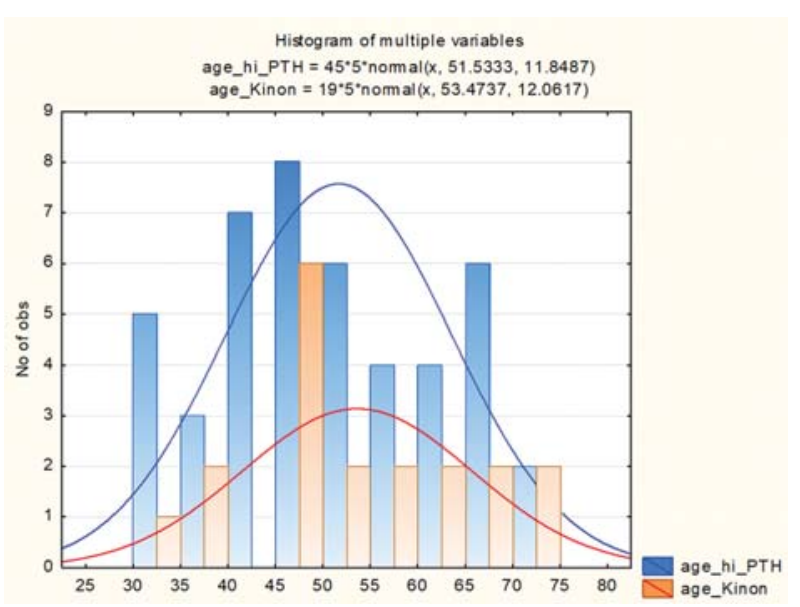

Fig. 4. BAP values in $H D$ patients

Tabl. 1. Dynamic change of PTH relative to sex

Frequency table: gender_hi_PTH

\section{Count}

Woman $(\operatorname{kod} 0)$

Men $(\operatorname{kod} 1)$

22

23
Cumulative - Count

22

45
Percent - of Valid Cumulative \% - of Valid

48.88889

48.8889

51.11111

100.0000
BAP is the bone-specific alkaline phosphatase isoform. A glycoprotein that is found on the surface of osteoblasts, BAP reflects the biosynthetic activity of these bone-forming cells. It has been shown to be a sensitive and reliable indicator of bone metabolism . Normal bones permanently undergo remodeling, where bone degradation or resorption is balanced by bone formation. If the process becomes unrelated and the rate of absorption exceeds the rate of formation, resulting in bone loss, it can lead to osteoporosis and therefore to higher fracture sensitivity.

High levels of BAP are associated with disability and high mortality in dialysis patients, emphasizing the dominant effect on bone turnover. BAP serum testing is of great importance for the monitoring of treatment in clinical practice in patients on dialysis treatment.

We have studied the levels of PTH in dialysis patients and patients at a pre-dialysis stage. Our results show a significant difference in serum BAP in the study groups of patients with high PTH and those with normal PTH (Fig. 4).

S. Sardiwal et al. (2013), (14) suggest the use of bone-specific alkaline phosphatase as an alternative to PTH because this enzyme is directly related to bone reorganization, reflects its histomorphometry and can predict the healing effects of hemodialysis in patients with CKD and disorders of the bone mineral metabolism. Bone-specific alkaline phosphatase hydrolyzes the pyrophosphate, which inhibits vessel calcification. Only bone-specific alkaline phosphatase is statistically significantly higher in patients with vascular calcifications than in the other patients $(\mathrm{p}<0.05)$. Multi-variance analysis demonstrated a statistically reliable association $(\mathrm{p}<0.0001)$ between the bone-specific alkaline phosphatase logarithm on the one hand and the calcification of the vessels (15).

In addition, high levels of serum phosphate inhibit the activity of 25-hydroxyvitamin D3-1ahydroxylase, the enzyme that converts vitamin $\mathrm{D}$ into its active form - calcitriol, thereby disrupting the CaSR and vitamin D receptor (VDR)-inverse feedback that suppresses PTH. As renal function decreases in CKD, FGF-23 levels also increase. This is due at least to the decreased klotho levels in the kidneys and the parathyroid gland, which occur when kidney function decreases $(16,17)$. 


\section{CONCLUSION}

In patients with $\mathrm{CKD}$, controlling PTH levels is recommended to keep them below $600 \mathrm{pg} / \mathrm{mL}$ to reduce the potential clinical consequences of SHPT as well as accompanying changes in calcium and phosphorus levels (KDIGO 2009).

Contemporary treatment aspects include vitamin D sterols and phosphate binders, which often prove insufficient to achieve the target $\mathrm{PTH}$, calcium and phosphorus levels (KDIGO 2009). Sometimes, the intake of vitamin $\mathrm{D}$ and phosphate binders is associated with a number of adverse events and challenges that may negatively affect patient adherence and persistence with regard to therapy (18).

Despite the widespread use of the metabolites of vitamin $\mathrm{D}$, phosphate binders and calcificers, in many patients with SHPT there is inadequate biochemical control. Consequently, the conclusion is that the lack of adherence and insufficient persistence in treatment increases the risk of failed therapy (19).

\section{REFERENCES}

1. Obi Y, Hamano T, Ichimaru N, Tomida K, Matsui I, Fujii N, et al. Vitamin D deficiency predicts decline in kidney allograft function: A prospective cohort study. J Clin Endocrinol Metab. 2014;99(2):527-35. doi: $10.1210 /$ jc.2013-2421.

2. Kilpatrick RD, Danese MD, Belozeroff V, Smirnakis K, Goodman WG, Rothman KJ. The association of vitamin D use with hypercalcemia and hyperphosphatemia in hemodialysis patients: a casecrossover study. Pharmacoepidemiol Drug Saf. 2011;20(9):914-21. doi: 10.1002/pds.2183.

3. Park H, Rascati KL, Lawson KA, Barner JC, Richards KM, Malone DC. Adherence and persistence to prescribed medicationtherapy among Medicare part $\mathrm{D}$ beneficiaries on dialysis: comparisons of benefit type and benefit phase. J Manag Care Spec Pharm. 2014;20(8):862-76. doi: 10.18553/ jmcp.2014.20.8.862.

4. Reams BD, Dluzniewski PJ, Do TP, Yue SV, Bradbury BD, Kshirsagar AV, et al. Dynamics of cinacalcet use and biochemical control inhemodialysis patients: a retrospective new-user cohort design. BMC Nephrol. 2015;16:175. doi: 10.1186/ s12882-015-0174-6.

5. Saliba W, El-Haddad B. Secondary hyperparathyroidism: pathophysiology and treatment. J Am
Board Fam Med. 2009;22(5):574-81. doi: 10.3122/ jabfm.2009.05.090026.

6. Mehrotra R, Kermah D, Budoff M, Salusky IB, Mao SS, Gao YL, et al. Hypovitaminosis D in chronic kidney disease. Clin J Am Soc Nephrol. 2008;3(4):1144-51. doi: 10.2215/CJN.05781207.

7. Danese MD, Belozeroff V, Smirnakis K, Rothman KJ. Consistent control of mineral and bone disorder in incident hemodialysis patients. Clin J Am Soc Nephrol. 2008;3(5):1423-9. doi: 10.2215/ CJN.01060308.

8. Block GA, Kilpatrick RD, Lowe KA, Wang W, Danese MD. CKD-mineral and bone disorder and risk of death and cardiovascular hospitalization in patients on hemodialysis. Clin J Am Soc Nephrol. 2013;8(12):2132-40. doi: 10.2215/CJN.04260413.

9. Cozzolino M, Tomlinson J, Walsh L, Bellasi A. Emerging drugs for secondary hyperparathyroidism. Expert Opin Emerg Drugs. 2015;20(2):197-208. doi: 10.1517/14728214.2015.1018177.

10. Ahmadi F, Damghani S, Lessan-Pezeshki M, Razeghi E, Maziar S, Mahdavi-Mazdeh M. Association of low vitamin D levels with metabolic syndrome in hemodialysis patients. Hemodial Int. 2016;20(2):261-9. doi: 10.1111/hdi.12316.

11. Liu GL, Pi HC, Hao L, Li DD, Wu YG, Dong J. Vitamin $\mathrm{D}$ status is an independent risk factor for global cognitive impairment in peritoneal dialysis patients. PLoS One. 2015;10(12):e0143782. doi: 10.1371/journal.pone.0143782.

12. Arbor Research Collaborative for Health: 2012 Annual Report of the Dialysis Outcomes andPractice Patterns Study: Hemodialysis Data 1997-2011. Available online: http://www.dopps.org/AnnualReport/(access date June 2015). 2012

13. Goodman WG, Quarles LD. Development and progression of secondary hyperparathyroidism inchronic kidney disease: lessons from molecular genetics. Kidney Int. 2008;74(3):276-88. doi: 10.1038/sj.ki.5002287.

14. Sardiwal S, Magnusson P, Goldsmith DJ, Lamb EJ. Bone alkaline phosphatase in CKD-mineral bone disorder. Am J Kidney Dis. 2013;62(4):810-22. doi: 10.1053/j.ajkd.2013.02.366.

15. Ishimura $E$, Okuno $S$, Okazaki $H$, Norimine K, Yamakawa K, Yamakawa T, et al. Significant association between bone-specific alkaline phosphatase and vascular calcification of the hand arteries 
Tracing and Comparing Serum, Specific, Bone Biomarkers in Patients with Secondary Hyperparathyroidism

in male hemodialysis patients. Kidney Blood Press

Res. 2014;39(4):299-307. doi: 10.1159/000355807.

16. Drueke TB, Massy ZA. Circulating Klotho levels: clinical relevance and relationship with tissue Klotho expression. Kidney Int. 2013;83(1):13-5. doi: 10.1038/ki.2012.370.

17. Moe SM, Thadhani R. What have we learned about chronic kidney disease-mineral bone disorder from the EVOLVE and PRIMO trials? Curr Opin Nephrol Hypertens. 2013;22(6):651-5. doi: 10.1097/ MNH.0b013e328365b3a3.

18. Heaney RP. Vitamin D in health and disease. Clin J Am Soc Nephrol. 2008;3(5):1535-41. doi: 10.2215/ CJN.01160308.

19. Bienaimé F, Girard D, Anglicheau D, Canaud G, Souberbielle JC, Kreis H, et al. Vitamin D status and outcomes after renal transplantation. J Am Soc Nephrol. 2013;24(5):831-41. doi: 10.1681/ ASN.2012060614. 